\title{
Perfil clínico de idosos atendidos em uma unidade de pronto atendimento em um hospital de Belém
}

\author{
Clinical profile of the elderly people assisted at an emergency care unit in a hospital in \\ Belém
}
Perfil clínico de los ancianos atendidos en una unidad de atención de emergencia en un hospital de Belém

Ana Karine Ribeiro Ximenes ${ }^{1}$, Tanise Nazaré Maia Costa ${ }^{1,2}$, Amanda Vallinoto Silva de Araújo ${ }^{2 *}$, Vânia Nazaré Maia dos Santos ${ }^{1}$, Matheus Sousa Alves².

\section{RESUMO}

Objetivo: Caracterizar o perfil clínico dos idosos que utilizaram o serviço de pronto atendimento de um hospital particular. Métodos: Este foi um estudo descritivo e retrospectivo em Belém - Pará, no primeiro semestre de 2017. Os dados foram coletados pela análise direta de 470 prontuários eletrônicos do hospital. A análise estatística foi realizada pelo software BioEstat $5.3 \AA$. A significância estatística foi aceita ao nível de $95 \%$ (pvalor < 0,05). Resultados: Quanto à classificação de risco de Manchester, a maioria dos idosos foi classificada como verde $(72,7 \%)$ e o diagnóstico mais frequente foram as infecções $(33,6 \%)$. Entre os idosos classificados como vermelho, o que mais motivou o atendimento foram as patologias de origem cardiovascular $(42,2 \%)$. A pneumonia $(22,2 \%)$ foi a principal causa de internação, porém, no geral, verificou-se uma baixa incidência de internações $(1,9 \%)$. Quanto ao desfecho clínico no pronto atendimento, observou-se um número significativo de altas $(99,4 \%)$ e um baixo percentual de óbito $(0,2 \%)$, de evasão $(0,2 \%)$ e de transferência $(0,2 \%)$. Conclusão: A maioria dos idosos atendidos foi da faixa etária entre 60 e 70 anos e do sexo feminino. Viu-se ainda que os processos infecciosos foram as que mais motivaram a busca pelo serviço.

Palavras-chave: Dinâmica populacional, Envelhecimento, Idoso, Epidemiologia, Emergência.

\begin{abstract}
Objective: To characterize the clinical profile of the elderly who used the emergency care service of a private hospital. Methods: This was a descriptive and retrospective study in Belém - Pará, in the first half of 2017. Data were collected by direct analysis of 470 electronic medical records at the hospital. Statistical analysis was performed using the BioEstat $5.3 \AA$ software. Statistical significance was accepted at the level of $95 \%$ ( $p$ $<0.05)$. Results: Regarding the Manchester risk classification, most elderly people were classified as green $(72.7 \%)$ and the most frequent diagnosis was infections (33.6\%). Among the elderly classified as red, the most motivating care was the pathologies of cardiovascular origin (42.2\%). Pneumonia (22.2\%) was the main cause of hospitalization, however, in general, there was a low incidence of hospitalizations (1.9\%). As for the clinical outcome in the emergency department, there was a significant number of discharges $(99.4 \%)$ and a low percentage of death $(0.2 \%)$, evasion $(0.2 \%)$ and transfer $(0.2 \%)$. Conclusion: Most of the elderly assisted were between 60 and 70 years old and female. It was also seen that the infectious processes were the ones that most motivated the search for the service.
\end{abstract}

Keywords: Population dynamics, Aging, Aged, Epidemiology, Emergencies.

\footnotetext{
1 Universidade do Estado do Pará (UEPA), Belém - PA. *E-mail: amandavsaraujo2@gmail.com

${ }^{2}$ Centro Universitário do Estado do Pará (CESUPA), Belém - PA.
} 


\section{RESUMEN}

Objetivo: caracterizar el perfil clínico de los ancianos que utilizaron el servicio de atención de emergencia de un hospital privado. Métodos: Este fue un estudio descriptivo y retrospectivo en Belém - Pará, en la primera mitad de 2017. Los datos fueron recolectados por análisis directo de 470 registros médicos electrónicos en el hospital. El análisis estadístico se realizó utilizando el software BioEstat 5.3®. La significancia estadística fue aceptada al nivel del 95\% ( $p<0.05$ ). Resultados: En cuanto a la clasificación de riesgo de Manchester, la mayoría de las personas mayores se clasificaron como verdes $(72.7 \%)$ y el diagnóstico más frecuente fue infecciones (33.6\%). Entre los ancianos clasificados como rojos, la atención más motivadora fueron las patologías de origen cardiovascular $(42,2 \%)$. La neumonía $(22,2 \%)$ fue la principal causa de hospitalización, sin embargo, en general, hubo una baja incidencia de hospitalizaciones (1,9\%). En cuanto al resultado clínico en el departamento de emergencias, hubo un número significativo de altas $(99.4 \%)$ y un bajo porcentaje de muertes $(0.2 \%)$, evasión $(0.2 \%)$ y transferencia $(0.2 \%)$ Conclusión: la mayoría de los ancianos asistidos tenían entre 60 y 70 años y eran mujeres. También se vio que los procesos infecciosos fueron los que más motivaron la búsqueda del servicio.

Palabras clave: Dinámica poblacional, Envejecimiento, Anciano, Epidemiologia, Urgencias medicas.

\section{INTRODUÇÃO}

O envelhecimento populacional é uma realidade nos países desenvolvidos. Dados publicados pela Organização Mundial de Saúde (OMS) mostram que a população com mais de 65 anos de idade aumentou de 5,61\% no ano 2000 para 7,90\% em 2015. O Relatório Mundial sobre o Envelhecimento (2015) afirma que a população com mais de 60 anos passará de $12,3 \%$ para $21,5 \%$ até 2050 (WORLD HEALTH ORGANIZATION, 2015).

No Brasil, segundo os dados do Instituto Brasileiro de Geografia e Estatística (IBGE), entre os anos de 2005 e de 2015 , a população de idosos passou de $9,8 \%$ para $14,5 \%$ do total da população brasileira. Este mesmo documento estima para o ano de 2050 que a população de idosos no Brasil será da ordem de 64 milhões, ou seja, corresponderá a $29,7 \%$ da população total (IBGE, 2016).

O critério cronológico é um dos mais utilizados para definir, conceituar o indivíduo como idoso, conceito este que é diferente entre os países desenvolvidos e os países em desenvolvimento. Nos primeiros, definição estabelecida pela Organização das Nações Unidas são consideradas idosas aquelas pessoas com 65 anos ou mais e nos segundos são idosas as pessoas com 60 anos ou mais. (SANTOS SSC, 2009).

Apesar de o critério cronológico ser o mais usado para conceituar o indivíduo idoso, este não é um marcador preciso das mudanças que acompanham o envelhecimento, pois este não ocorre de forma homogênea, permitindo que cada indivíduo vivencie isso de uma forma muito particular já que o processo de envelhecimento provoca no organismo modificações biológicas, psicológicas e sociais (SANTOS SSC, 2009).

Segundo Minaker K (2009), as principais modificações biológicas que ocorrem com o envelhecimento, entre outras, são Sistema cardiovascular, o espessamento e endurecimento da parede ventricular esquerda, predispondo a insuficiência cardíaca diastólica; há perda das células do marcapasso do nó sinusal, promovendo redução da frequência cardíaca e deixando o idoso mais suscetível a arritmias; dilatação da aorta e enrijecimento de suas paredes, provocando um aumento da pressão arterial sistólica.

Já no sistema respiratório: o enrijecimento da parede torácica leva a uma combinação de ventilação/perfusão malsucedida, com redução de $50 \%$ na capacidade das trocas alveolares, a princípio sem repercussões, mas que em situações de estresse pode causar fadiga e falta de ar. Reflexos de tosse e função ciliar também diminuem, promovendo acúmulo de secreções e predispondo a infecções; dentre outros sistemas que são afetados e que podem comprometer a qualidade de vida do paciente idoso.

Esse declínio funcional deixa os idosos mais suscetíveis a determinadas condições que são denominadas "síndromes geriátricas" ou, como também são conhecidas, os cinco "is" da geriatria. São elas: imobilidade, 
instabilidade postural, insuficiência cognitiva, incontinência urinária, insuficiência familiar e, mais recentemente nesse contexto, foi incluída a "síndrome da fragilidade", que consiste em desregulação neuroendócrina, disfunção imunológica e sarcopenia (DUARTE Y, 2018).

Diante do exposto, não causa estranhamento que esse indivíduo idoso seja uma presença frequente nos serviços de emergência. É analisado que os idosos quando comparados aos adultos jovens, em geral, necessitam de um atendimento emergencial com mais frequência e de uma quantidade maior de recursos (medicações, exames complementares), permanecem mais tempo nas unidades de emergência e têm o dobro de chances de internação nas mesmas condições e que, por isso, é muito importante reconhecer as demandas especiais dessa população (PATRICIO ACFA, et al., 2016).

O processo de transição demográfica, com o crescente número de idosos, trouxe também mudanças no perfil das doenças. Doenças infecciosas, que antes eram as principais causas de óbito na população, foram substituídas por doenças crônicas não transmissíveis, cada vez mais prevalentes. Atualmente, no Brasil, é marcante o predomínio de doenças crônicas; porém, em determinados momentos, ainda é possível perceber uma superposição entre estas e as doenças infectocontagiosas (DUARTE EC, et al., 2012).

Os idosos são a parcela da população mais sujeita às doenças cardiovasculares, às doenças respiratórias - como a Doença Pulmonar Obstrutiva Crônica (DPOC) - e aos acidentes vasculares cerebrais, por causas orgânicas ou mesmo por trauma, uma vez que as quedas costumam ser comuns neste grupo. Também estão mais expostos a intoxicações e a eventos adversos de medicamentos, relacionados a iatrogenia (MINAKER K, 2010).

Segundo as Diretrizes para o Cuidado da Pessoa Idosa (2014) o declínio funcional do idoso ocorre em um cenário de múltiplos e complexos problemas de saúde, de modo que o perfil epidemiológico da população idosa se caracteriza pela tripla carga de doenças com forte predomínio das condições crônicas, prevalência de elevadas mortalidade e morbidade por condições agudas e complicações de condições crônicas (BRASIL, 2014).

Uma pesquisa também cita as condições crônicas mais prevalentes nos idosos, a hipertensão arterial e a destacam como o principal fator de risco para as doenças cardiovasculares e cerebrovasculares, que por sua vez estão entre as principais causas de óbito e de incapacidade funcional entre os idosos. Estas autoras ainda ressaltam as doenças osteoarticulares como uma causa importante de incapacidade funcional no idoso e que, entre outros fatores, também é um fator de risco importante para um evento drástico na vida do idoso que são as quedas (RODRIGUES CC, et al., 2012).

Conhecer o perfil clínico-epidemiológico desse idoso, que com frequência busca atendimento nos serviços de emergência, possibilita um reconhecimento mais precoce das etiologias mais comuns nesse grupo, favorecendo um diagnóstico mais precoce e assertivo, quiçá reduzindo o número procedimentos desnecessários e minimizando possíveis iatrogenias e piores desfechos.

O objetivo deste trabalho foi traçar o perfil clínico dos idosos atendidos em uma unidade de pronto atendimento de um hospital privado de modo a quantificar o número total de idosos atendidos, analisar quanto à frequência neste pronto atendimento, às variáveis diagnóstico e comorbidades e verificar o desfecho clínico (alta/óbito) no período determinado.

\section{MÉTODOS}

Trata-se de um estudo retrospectivo e descritivo-analítico, baseado na análise quantitativa de dados contidos nos prontuários eletrônicos dos pacientes idosos atendidos de janeiro a junho de 2017, na unidade de pronto atendimento (PA) de um Hospital de Belém - Pará. O projeto foi aprovado pelo Comitê de Ética em Pesquisa sob número de parecer 2.460.442.

A partir de um formulário próprio dos autores, foram retiradas informações relativas à classificação de risco, diagnóstico de admissão no pronto atendimento (PA), comorbidades, número e causas de óbito e número de altas. Do total de idosos atendidos no período em questão, foi selecionada de forma randomizada 
uma amostra de 470 prontuários, que visou a minimizar os vieses associados à sazonalidade de determinadas patologias. Foram excluídos, por sua vez, todos os indivíduos com idade inferior a 60 anos e que não foram atendidos no período estipulado. Este trabalho foi submetido ao Comitê de Ética em Pesquisa, sendo iniciado após a sua aprovação.

A estatística analítica foi utilizada para avaliar os resultados das variáveis amostrais através dos Testes $G$ e Qui-Quadrado Aderência para tabelas univariadas, Testes G e Qui-Quadrado Independência para tabelas bivariadas e Teste Qui-Quadrado Partição para a comparação bivariada com mais de duas categorias. As estatísticas descritiva e analítica foram realizadas no software BioEstat@ 5.3 (6). Para a tomada de decisão, adotou-se o nível de significância $\alpha=0,05$ ou $5 \%$.

\section{RESULTADOS}

No período de janeiro a junho de 2017, foram atendidos um total de 9.338 idosos no PA. Em se tratando das patologias que motivaram os idosos a buscarem o PA, as infecções foram responsáveis por $33,6 \%$ dos casos, com uma incidência significante $(p=0,0006)$. As doenças osteoarticulares e musculares foram responsáveis por $21,7 \%$ e, em terceiro lugar, a maior incidência foi daquelas denominadas de "outras causas", com $13,6 \%$. Sob esta denominação ficaram os casos mais esporádicos, como cefaleia, fadiga, "mal-estar", alergias, insônia, quadros ansiosos, alguns retornos pós-cirúrgicos, ou mesmo realização de procedimento (troca de cateter vesical, por exemplo).

As doenças do aparelho digestivo (gastrite, esofagite, dispepsia), assim como as quedas de qualquer caráter foram responsáveis cada uma por 7,02\% dessa amostra, e as doenças cardiovasculares por 5,95\% dos casos, figurando em 5임 lugar (Tabela 1).

Tabela 1 - Principais afecções diagnosticadas entre os idosos atendidos no pronto atendimento de um hospital privado de Belém/PA.

\begin{tabular}{lll}
\hline Principais afecções & Freq & $\%$ \\
\hline Infecções & 158 & $33,6 \%$ \\
Doença Osteoarticular e Muscular & 102 & $21,7 \%$ \\
Doença Aparelho Digestivo & 33 & $7,0 \%$ \\
Queda & 33 & $7,0 \%$ \\
Doença Cardiovascular & 28 & $6,0 \%$ \\
Doença Aparelho Pulmonar & 12 & $2,6 \%$ \\
Doença Aparelho Urológico & 9 & $1,9 \%$ \\
Doença Oncológica & 6 & $1,3 \%$ \\
DM descompensada & 2 & $0,4 \%$ \\
Doença Cerebrovascular & 2 & $0,4 \%$ \\
Outras causas & 64 & $13,6 \%$ \\
Sem diagnóstico & 21 & $4,5 \%$ \\
\hline Total & 470 & $100,0 \%$ \\
\hline
\end{tabular}

Fonte: Ximenes AKR, et al., 2020. Banco de dados do hospital (sistema Tasy®).

É possível verificar que entre as infecções, as de vias aéreas superiores (IVAS) foram as de maior destaque, totalizando $41,77 \%$, seguidas pelos casos de gastroenterocolite aguda (GECA), respondendo a $27,84 \%$ das infecções $(p<0,00001)$ (Figura 1). 
Figura 1 - Infecções mais incidentes entre os idosos atendidos no pronto atendimento de um hospital particular de Belém/Pa.

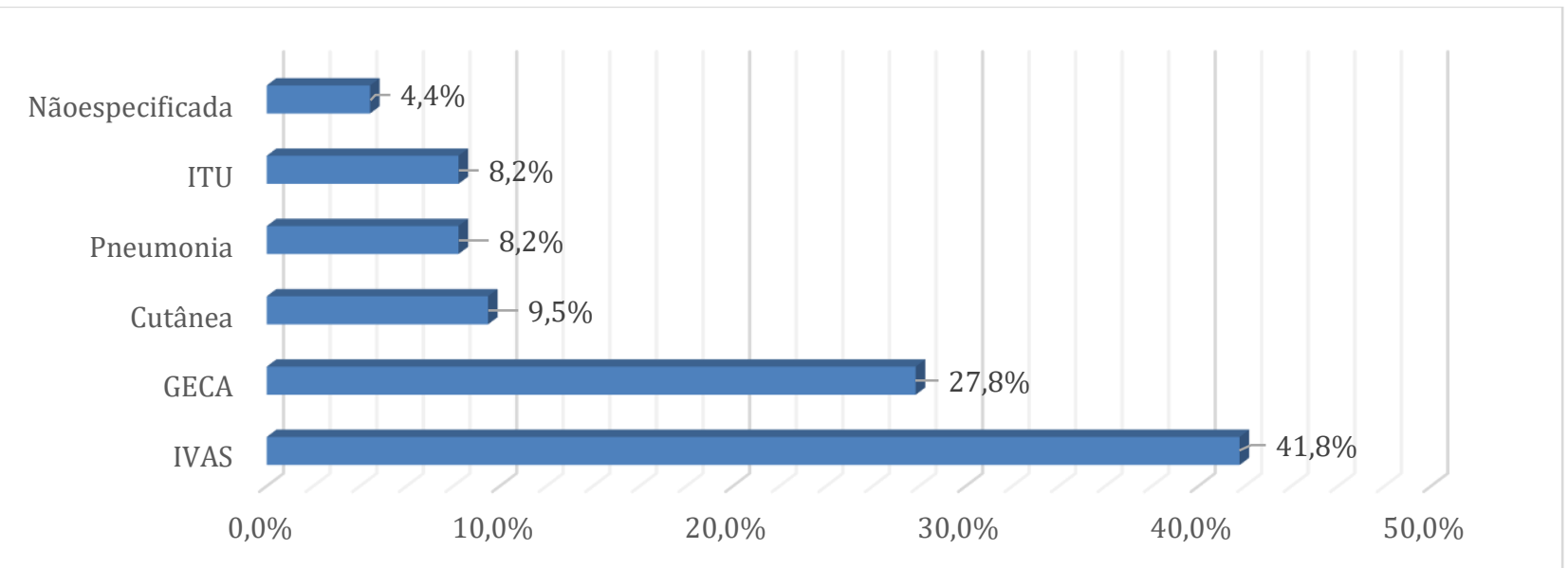

Fonte: Ximenes AKR, et al., 2020.

Analisando a população de idosos classificados como emergência (vermelho), observou-se que as doenças cardiovasculares figuraram no topo dessa lista, respondendo a $42,2 \%$ desta população, seguidas pelas doenças cerebrovasculares com 10,9\% e, em terceiro lugar, não menos importantes, ficaram as quedas de qualquer natureza, com um percentual de $7,8 \%(p<0,0001)$.

Entre as principais causas de doenças cardiovasculares estão as urgências/emergências hipertensivas e as queixas de dor torácica não especificadas, além das arritmias e do infarto agudo do miocárdio (IAM), nessa sequência (Figura 2).

Figura 2 - Etiologias clínicas mais incidentes entre a população total de idosos classificados como emergência (vermelho), atendidos no pronto atendimento de um hospital privado de Belém/PA.

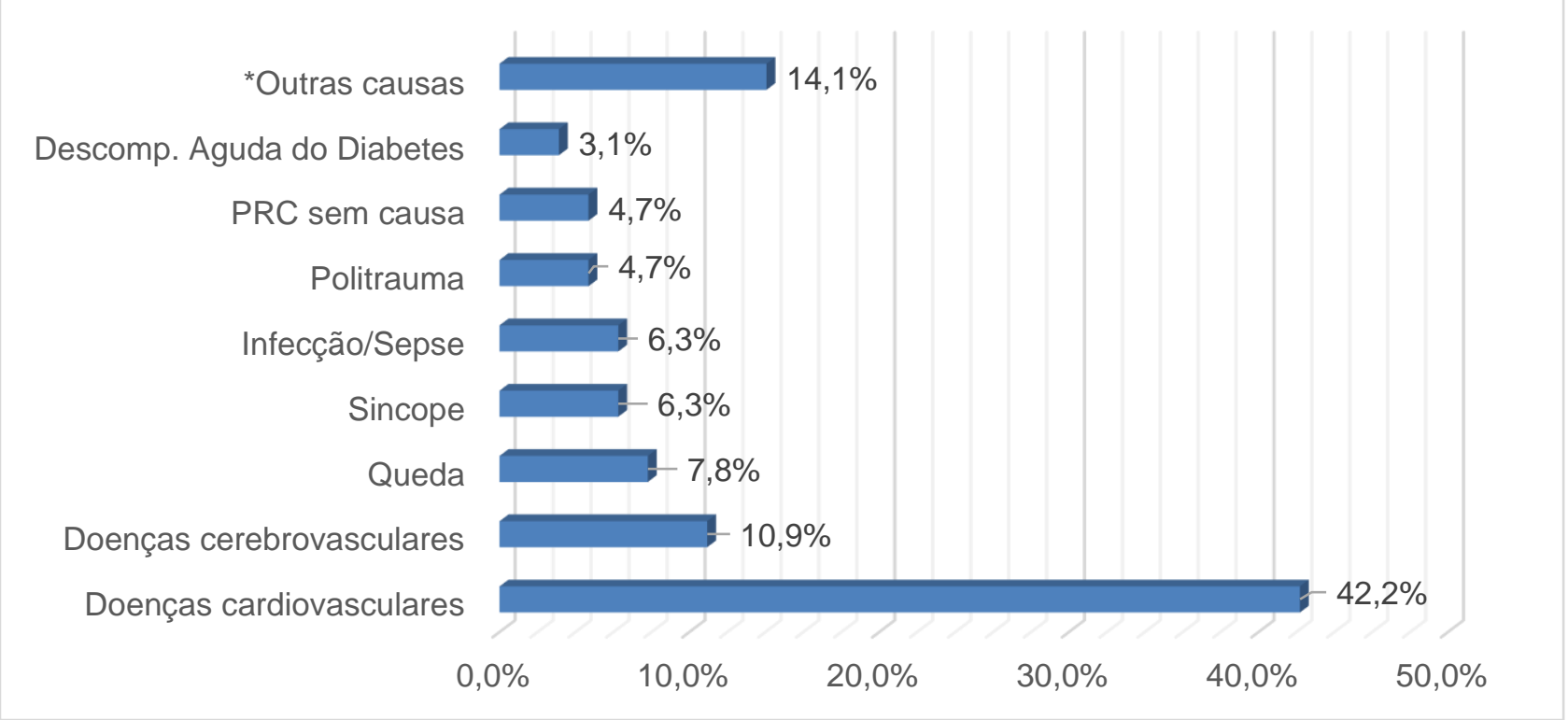

Legenda: *Outras causas: convulsão, alergias, desnutrição, cefaleia...). Fonte: Ximenes AKR, et al., 2020.

$\mathrm{Na}$ amostra avaliada de 470 prontuários, verificou-se que em relação a comorbidades, mais da metade dos idosos $(65,3 \%)$ apresentavam ao menos um tipo. A hipertensão arterial sistêmica (HAS) foi a mais prevalente, respondendo a $78,8 \%$, seguida pelo diabetes mellitus (DM), com $35,5 \%$ e pelas cardiopatias, com $18,6 \%(p<0,00001)$. A associação entre a HAS e o DM foi a mais comumente encontrada (Tabela 2$)$. 
Tabela 2 - Comorbidades mais prevalentes entre os idosos atendidos em um pronto atendimento de um hospital privado de Belém/PA.

\begin{tabular}{ccc}
\hline Comorbidades & Frequência & $\%$ \\
\hline Presença & & \\
\hline Sim & 307 & $65,3 \%$ \\
Não & 163 & $34,7 \%$ \\
\hline Tipos & 242 & $\mathrm{~N}=307$ \\
\hline HAS & 109 & $78,8 \%$ \\
Diabetes Mellitus & 57 & $35,5 \%$ \\
Cardiopatia & 16 & $18,6 \%$ \\
Asma/DPOC & 16 & $5,2 \%$ \\
Gastrite & 12 & $5,2 \%$ \\
Hipotireoidismo & 12 & $3,9 \%$ \\
Artrose/Osteoporose & 9 & $3,9 \%$ \\
Demência & 3 & $2,9 \%$ \\
DRC & & $1,0 \%$ \\
\hline
\end{tabular}

Fonte: Ximenes AKR, et al., 2020.

De acordo com a amostra analisada, 1,9\% (09) dos idosos atendidos evoluíram para internação. Entre estes a principal etiologia que motivou a internação foi a pneumonia, a qual foi a principal com $22,2 \%$ (Tabela 3).

Tabela 3 - Causas de internação entre os idosos atendidos no pronto atendimento de um hospital privado de Belém/PA.

\begin{tabular}{ccc}
\hline Internação dos idosos & Frequência & $\%$ \\
\hline Ocorrência & & \\
\hline Sim & 9 & $1,9 \%$ \\
Não & 461 & $98,1 \%$ \\
\hline Causa da internação & & $\mathbf{N = 0 9}$ \\
\hline Pneumonia & 2 & $22,2 \%$ \\
Sepse & 1 & $11,1 \%$ \\
Hemorragia digestiva & 1 & $11,1 \%$ \\
AVC Agudo & 1 & $11,1 \%$ \\
Dor torácica NE & 1 & $11,1 \%$ \\
Queda/Fratura & 1 & $11,1 \%$ \\
Dor abdominal NE & 1 & $11,1 \%$ \\
IC descompensada & 1 & $11,1 \%$ \\
\hline
\end{tabular}

Fonte: Ximenes AKR, et al., 2020.

Com relação ao desfecho clínico, dos 470 pacientes que foram atendidos no PA, incluindo os que necessitaram de internação, $467(99,4 \%)$ receberam alta hospitalar. Nesta amostra foi observado apenas um caso de óbito $(0,2 \%)$ na emergência. Este já chegou em parada cardiorrespiratória de causa indeterminada. Foi observado apenas um caso de evasão $(0,2 \%)$ e um caso de transferência para outro hospital $(0,2 \%)$ (Tabela 4).

Tabela 4 - Desfecho clínico entre os idosos atendidos no pronto atendimento de um hospital privado de Belém/PA.

\begin{tabular}{ccc}
\hline Desfecho clínico & Freq & $\%$ \\
\hline Alta* & 467 & $99,4 \%$ \\
Óbito & 1 & $0,2 \%$ \\
Transferência & 1 & $0,2 \%$ \\
Evasão & 1 & $0,2 \%$ \\
\hline Total & 470 & $100,0 \%$ \\
\hline
\end{tabular}

Fonte: Ximenes AKR, et al., 2020. 


\section{DISCUSSÃO}

De janeiro a junho de 2017, foram atendidos neste PA um total de 9.338 idosos. As infecções $(33,6 \%)$, principalmente as de vias aéreas superiores $(41,8 \%)$, foram as mais incidentes nesta pesquisa, seguidas pelas doenças do aparelho osteomuscular $(21,7 \%)$, o que justifica inclusive o número de pacientes classificados como verdes.

Isso é justificável por características inerentes ao processo de envelhecimento, como o declínio da função imune e as alterações do sistema musculoesquelético, predispondo inclusive esse idoso à síndrome de fragilidade e a quedas (MINAKER K, 2010; AYRES M, et al., 2007). Esse percentual representativo de idosos atendidos só reforça o cenário de envelhecimento demográfico e que os serviços de saúde deverão se preparar para essa nova demanda. O Instituto Brasileiro de Geografia e estatística (IBGE), em 2016, estima que a população de idosos no Brasil em 2070 (35\%) será superior à dos países desenvolvidos e que essa transição ocorrerá de forma acelerada.

Entre os principais fatores de risco para as doenças cardiovasculares, a Hipertensão Arterial (HA) e o Diabetes Mellitus (DM) são as principais comorbidades encontradas, atingindo mais de $60 \%$ nos idosos (MALACHIAS MV, et al., 2016). Nesta pesquisa, mais da metade dos idosos da amostra $(65,3 \%)$ apresentavam no mínimo um tipo de comorbidade e, entre elas, a HA $(78,8 \%)$ e o DM $(35,5 \%)$ foram as mais prevalentes entre os idosos, assemelhando-se aos resultados encontrados na literatura $(8,9)$. A prevalência de HA e do DM é crescente conforme a idade, e, quando associadas, elevam consideravelmente a morbimortalidade (XAVIER CM, 2014; CUNHA BSS, et al., 2014; FRANCISCO PMSB, et al., 2018). Diante deste cenário, justifica-se a maior frequência de atendimentos de doenças cardiovasculares (42,2\%) e cerebrovasculares $(10,9 \%)$ encontradas nesta pesquisa, assim como seu potencial de gravidade, pois foram identificadas inclusive entre os idosos classificados como vermelho (emergência).

Dados do Instituto Brasileiro de Geografia e Estatística (2016) (IBGE, 2016) reforçam essa ideia quando afirma que as principais causas de mortalidade entre idosos se concentram nas doenças do aparelho circulatório $(36,3 \%)$, seguidas pelas neoplasias $(18,6 \%)$ e as doenças do aparelho respiratório $(15,5 \%)$.

Ainda assim, as quedas representaram $7,8 \%$ do percentual total. Em idosos da comunidade a prevalência de queda varia entre $10 \%$ e $40 \%$ e sendo um evento potencialmente catastrófico na vida do idoso e sua ocorrência relacionada a altas taxas de morbimortalidade é importante que os profissionais que trabalham nas emergências estejam atentos para essa situação e que se faça, ainda que minimamente, uma investigação das possíveis causas dessas quedas no âmbito da emergência para evitar recorrências. (OLIVEIRA AS, et al., 2014).

Embora o envelhecimento seja um processo heterogêneo, é fato que com o passar dos anos há uma redução das reservas orgânicas e funcionais que, somadas às lesões de órgãos-alvo provocadas por doenças crônico-degenerativas, contribuem para que estes indivíduos cheguem à emergência em condições clínicas de maior risco e demandando maior atenção (FREITAS EV, et al., 2016)

Ainda entre os idosos que foram classificados como vermelhos, as quedas representaram $7,8 \%$ do percentual total. Em idosos da comunidade a prevalência de queda varia entre $10 \%$ e $40 \%$ e sendo um evento potencialmente catastrófico na vida do idoso e sua ocorrência relacionada a altas taxas de morbimortalidade é importante que os profissionais que trabalham nas emergências estejam atentos para essa situação e que se faça, ainda que minimamente, uma investigação das possíveis causas dessas quedas no âmbito da emergência para evitar recorrências (OLIVEIRA AS, et al., 2014)

Analisando apenas a população total de idosos distribuída por faixa etária e levando em consideração a classificação de risco, fica evidente uma maior concentração de idosos entre 60 e 70 anos, responsável por $56,6 \%$ (5.288). Silva EC (2016) obteve resultado semelhante, evidenciando que os idosos entre 60 e 69 anos foram os que mais procuraram uma unidade de pronto atendimento (UPA) no município de Maringá/PR, assim como na pesquisa de Cunha, Nascimento e Sá (2014), realizada na emergência de um hospital federal do Rio de Janeiro. Uma vez que a expectativa de vida no Brasil se encontra em torno de 75,5 anos (IBGE, 2016), é natural que haja uma maior concentração de idosos jovens (60-70 anos). 
$\mathrm{Na}$ avaliação dessa amostra, apenas 1,9\% dos idosos evoluíram com necessidade de internação, número esse que foi bem inferior a taxa de internação do próprio hospital onde foi realizada esta pesquisa, que varia entre $8 \%$ e $9 \%$. Este é um dado que diverge da literatura, na qual se observa um maior número de internação entre os idosos e um tempo de internação mais prolongado (VERAS RO, 2009).

Inclusive o risco de internação aumenta acentuadamente com a idade: de $11,8 \%$ aos idosos com 60 a 69 anos para $17,7 \%$ aos idosos com a faixa etária de 70 a 79 anos e $24,2 \%$ aos idosos acima de 80 anos (LOYOLA FAl, et al., 2004). Esse viés pode estar associado ao fato de que esta pesquisa avaliou atendimentos pontuais no pronto atendimento e que naquele momento não necessitaram de internação, porém podendo ter evoluído para internação em um atendimento posterior.

A partir de uma amostra de 470 prontuários, verificou-se que o maior número percentual de atendimentos no pronto atendimento (PA) deste hospital foi o do sexo feminino $(64,46 \%)$, que foi diferente do encontrado em outras pesquisas como a de).

Em ambas a maior procura pelo serviço de pronto atendimento se deu pelo sexo masculino com 52,06\% e 51,8\%, respectivamente. Apenas Xavier CM (2014) encontrou resultado semelhante a esta pesquisa, com um maior número de mulheres atendidas na emergência $(58,52 \%)$ e com predomínio de idosas (RODRIGES CC, 2012; CUNHA BSS, 2014).

A menor procura dos homens pelos serviços de saúde está relacionada a um modelo hegemônico de masculinidade, no qual este homem se vê como invulnerável e vendo-se assim se expõe mais e se torna mais vulnerável, de tal forma que este só procura os serviços de saúde em situações de maior gravidade (GOMES $\mathrm{R}$, et al., 2007).

Nesse contexto, a pneumonia foi a principal causa de internação entre os idosos, o que também divergiu do encontrado na literatura, que aponta as doenças do aparelho circulatório como a principal causa de internação de pacientes idosos no Brasil (SILVA EC, et al., 2016). Porém, em um estudo Xavier CM (2014), as doenças associadas ao aparelho respiratório (incluindo a pneumonia) foram as principais causas de internação para adultos de todas as idades, inclusive entre os idosos.

Avaliando o desfecho clínico, dos 470 pacientes que foram atendidos nessa emergência $99,4 \%$ receberam alta, apenas um óbito $(0,2 \%)$ foi evidenciado na emergência e por causa desconhecida. Acredita-se que isto se justifique pela própria característica do paciente atendido nesta emergência, pacientes que em sua maioria foram classificados como verdes (pouco urgente), idosos considerados jovens, ou seja, em melhor condição clínica e teoricamente com uma melhor reserva funcional. Alia-se a isso, o fato de que a sobrevida do paciente crítico depende de um atendimento inicial, rápido e eficaz (SILVA EC, et al., 2016).

$\mathrm{Na}$ amostra em questão, houve um pequeno número que evoluiu com necessidade de internação, o que talvez não reflita de fato a realidade, uma vez que a taxa de internação do próprio hospital é bem mais alta. É provável que alguns possam ter se convertido para internação em outro momento não captado nesta pesquisa. Na amostra estudada, em relação ao desfecho clínico, mais de $90 \%$ dos pacientes evoluíram para alta hospitalar, demonstrando que a presteza no atendimento faz toda a diferença na evolução do quadro. As particularidades inerentes ao envelhecimento podem aumentar a gravidade das doenças e o risco de mortalidade dessa população, sendo necessários conhecimentos geriátricos e específicos.

Conclui-se, portanto, que é necessária uma assistência voltada aos idosos, com o objetivo de minimizar iatrogenias, reduzir taxar de internação hospitalar e, assim, promover um melhor atendimento à essa crescente parcela da população.

\section{CONCLUSÃO}

Os resultados desta pesquisa evidenciaram que a maioria dos idosos atendidos foi da faixa etária entre 60 e 70 anos (idoso jovem) e do sexo feminino. Viu-se ainda que os processos infecciosos, com destaque para as infecções de vias aéreas superiores, foram a que mais motivaram a busca pelo serviço. Em se tratando do grupo de idosos que foram classificados como vermelhos e receberam atendimento de emergência, as 
condições que mais motivaram o atendimento foram as doenças cardiovasculares e cerebrovasculares. Este ponto se aproxima muito dos resultados de outras pesquisas realizadas, inclusive em hospitais públicos. Com isso, torna-se evidente a necessidade de maior atenção, independentemente da condição financeira, ao controle de fatores de risco, como a HAS e o DM, que inclusive foram as comorbidades mais prevalentes neste estudo. Quanto às internações, a principal causa foi por pneumonia.

\section{REFERÊNCIAS}

1. AYRES M, et al. Bioestat 5.0 aplicações estatísticas nas áreas das ciências biológicas e médicas. Belém: ONG Mamiraua; 2007. 364p.

2. BRASIL. Ministério da Saúde. Diretrizes para o cuidado das pessoas idosas no SUS: proposta de modelo de atenção integral. Ministério da Saúde: Brasília; 2014.

3. CUNHA BSS, et al. Perfil Clínico e Sóciodemográfico de Internação de Idosos na Unidade de Emergência de um Hospital Geral. Estudos Interdiciplinares sobre o Envelhecimento. 2014; 19 (1): 198-200.

4. DUARTE EC, et al. Transição demográfica e epidemiológica: a Epidemiologia e Serviços de Saúde revisita e atualiza o tema. Epidemiol. Serv. Saúde [Internet]. 2012; 21(4): 529-532.

5. DUARTE Y. Grandes Síndromes Geriátricas. [2018]. Disponível em: <https://edisciplinas.usp.br/pluginfile.php/4111269/mod_resource/content/0/Texto\%20de\%20apoio_Sindromes\%20g eriátricas.pdf>.

6. FRANCISCO PMSB, et al. Prevalência simultânea de hipertensão e diabetes em idosos brasileiros: desigualdades individuais e contextuais. Cien Saude Colet [Internet]. 2018; 23 (11): 3829-3840.

7. FREITAS EV, et al. Tratado de Geriatria e Gerontologia.4 ed, Rio de Janeiro: Guanabara Koogan, 2016.

8. GOMES R, et al. Por que os homens buscam menos os serviços de saúde do que as mulheres? As explicações de homens com baixa escolaridade e homens com ensino superior. Cad. Saúde Pública. 2007, 23(3): 565-574.

9. INSTITUTO BRASILEIRO DE GEOGRAFIA E ESTATÍSTICA (IBGE). Síntese de indicadores sociais: uma análise das condições de vida da população brasileira: 2016/IBGE [Internet]. Estudos e pesquisas. Informação demográfica e socioeconômica. Rio de Janeiro: IBGE; 2016, 36:1516-3296

10. LOYOLA FAI, et al. Causas de internações hospitalares entre idosos brasileiros no âmbito do Sistema Único de Saúde. Epidemiol. Serv. Saúde [Internet]. Dez 2004; 13 (4): 229-238.

11. MALACHIAS MV, et al. Sociedade Brasileira de Cardiologia; Sociedade Brasileira de Hipertensão; Sociedade Brasileira de Nefrologia. 7ª Diretriz Brasileira de Hipertensão Arterial. Arq Bras Cardiol [Internet]. 2016,107 (3 Suppl 3):1-83.

12. MINAKER K Sequelas clínicas comuns do envelhecimento. In: Goldman L, Ausiello, D. Cecil - Tratado de medicina interna. 23 ed. (Elsevier). Rio de Janeiro; 2010: 128-134.

13. OLIVEIRA AS, et al. Fatores ambientais e risco de quedas em idosos: revisão sistemática. Rev. Bras. Geriatr. Gerontol [Internet]. 2014; 17 (3): 637-645.

14. PATRICIO ACFA. et al. Atendimento pré-hospitalar móvel: identificando agravos à saúde da pessoa idosa. Rev. de Pesq. Cuidado é Fundamental. 2016: 4223-4230.

15. RODRIGUES CC, et al. Perfil epidemiológico dos idosos atendidos na emergência de um hospital escola. Arquivos de Ciências da Saúde. São José do Rio Preto. 2012, 19: 37-41.

16. SANTOS SSC. Concepções teórico-filosóficas sobre envelhecimento, velhice, idoso e enfermagem gerontogeriátrica. Rev. Bras. Enf. 2010, 63(6): 1035-1039.

17. SILVA EC, et al. Classificação de risco de Idosos Atendidos em uma Unidade de Urgência e Emergência. Enciclopédia Biosfera, Centro Científico Conhecer [Internet]. 2016; 13 (23): 1467-1478.

18. XAVIER CM. Perfil da Demanda de Emergências Clínicas no Pronto Socorro do Hospital Regional da Ceilândia-DF. Brasília [bacharelado em enfermagem] -Universidade de Brasília; 2014.

19. WORLD HEALTH ORGANIZATION. World report on ageing and health. Geneva: WHO Library Cataloguing-inPublication Data; 2015.

20. VERAS RO. Envelhecimento populacional contemporâneo: demandas, desafios e inovações. Rev. Saúde Pública [Internet]. Jun 2009; 43 (3): 548-554. 